\title{
A Strong Force Potential Formula and the Classification of the Strong Interaction
}

\author{
Zhengdong Huang \\ Hubei Zhouheiya Enterprise Development Co., Ltd., Wuhan, China \\ Email:634821261@qq.com
}

How to cite this paper: Huang, Z.D. (2018) A Strong Force Potential Formula and the Classification of the Strong Interaction. Open Access Library Journal, 5: e4187.

https://doi.org/10.4236/oalib.1104187

Received: November 23, 2017

Accepted: January 26, 2018

Published: January 29, 2018

Copyright (๐ 2018 by author and Open Access Library Inc.

This work is licensed under the Creative Commons Attribution International License (CC BY 4.0).

http://creativecommons.org/licenses/by/4.0/

\section{(c) (i) Open Access}

\begin{abstract}
The most difficult problem in the research on strong interaction is to solve the mechanical expression of strong nuclear force, thus failing to comprehensively describe the state in and between hadrons. In this paper, reasonable strong force potential hypothesis is proposed through analysis, and strong interaction is classified into 5 types according to different stress particles; then, different internal symmetrical structures are correspondingly obtained, and Dirac equation is adopted to describe the intrinsic states of 13 particles of 5 types; finally, the theoretical value and the experimental value of the mass ratio of the same-charged particles and the neutral particles are compared to prove the complete set of research method and verify the correctness of the strong force potential formula supposed thereby.
\end{abstract}

\section{Subject Areas}

Particle Physics

\section{Keywords}

Strong Interaction, Strong Nuclear Force, Strong Force Potential, Mass Ratio

\section{Introduction}

\subsection{Classification of Strong Interaction}

\subsubsection{History of Strong Force Research and Solution of Pending Problem} Strong interaction is an action in or between hadrons. Strong force maintains the stability of internal structure of hadrons and the interaction between hadrons, thus contributing to the enrichment of particles family. However, people have not yet found the suitable breakthrough point for studying the essence of strong interaction. Many strong interaction processing theories and methods proposed in the past only have limited achievements, and the mechanical ex- 
pression as perfect as that of gravitational force and electromagnetic force has not yet been found till now.

The author believes that the key for solving the strong interaction problem is to positively solve strong force potential, and the suitable mathematical toolDirac equation can be taken as the direct evidence to prove the mass ratio of the same-charged particles and neutral particles ("charged" mentioned in this paper means charge-carrying).

\subsubsection{Five Types of Strong Interaction}

Strong interaction can be divided into five types according to different stress particles.

1) Baryon type strong interaction in particles;

2) Meson type strong interaction in particles;

The stress particles for the two types of basic strong interaction in hadrons are quarks, but it does not mean that the stress particles for all types of strong interaction are quarks, because strong interaction can be also generated between hadrons. Therefore, besides the above two types of basic strong interaction, another three types of strong interaction are also included.

3) Strong interaction between baryons is called as inter-baryon type strong interaction, and the representative particles include deuteron [1], triton [1] and atomic nucleuses thereof;

4) Strong interaction between baryon and meson is called as baryon-meson type strong interaction, and the representative particles include $\Delta++$ (1232) [2], $\Delta+$ (1232) [2], $\Delta 0$ (1232) [2] and $\Delta-$ (1232) [2];

5) Strong interaction between mesons is called as inter-meson type strong interaction, and the representative particles include $\eta$ [3] particle and $\eta^{\prime}$ [3] particle.

For the three types of strong interaction based on the two types of basic strong interaction, the action particles thereof are hadrons (internal structure particles of hadrons are quarks), so such strong interaction is also called as combined type strong interaction.

\subsubsection{Internal Symmetrical Structure of Hadrons}

Two factors can influence the internal symmetrical structures of hadrons, namely: parity of quantity of stress particles and mass difference of stress particles. Hadrons have two types of internal symmetrical structures. Specifically, one is the plane symmetrical structure, and such structure can be further divided into odd symmetrical structure (as shown in Figure 1(a) and Figure 1(e)) and even symmetrical structure (as shown in Figure 1(b) and Figure 1(c)), and it is the most basic, general and stable symmetrical structure in hadrons; the other one is spatial symmetrical structure (as shown in Figure 1(d)), and such structure is rarely seen, and the representative particles include $\eta$ particle and $\eta^{\prime}$ particle.

Baryon type strong interaction can be further divided into baryon type odd 


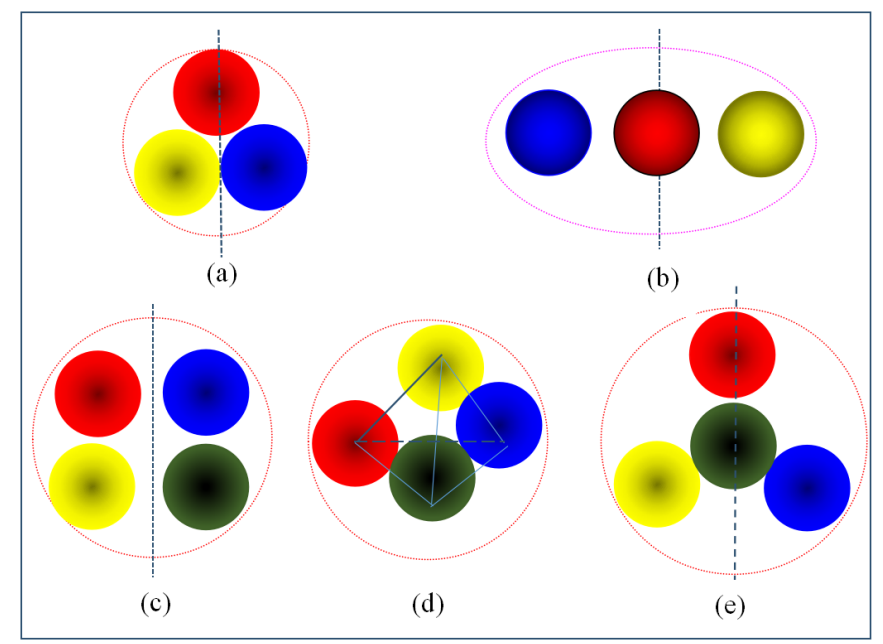

Figure 1. (a) Internal odd symmetrical structure diagram of force-carrying particle ( $\mathrm{n}=$ 3); (b) Internal even symmetrical structure diagram of force-carrying particle ( $\mathrm{n}=3)$; (c) Internal Even symmetrical structure diagram of force-carrying particle $(n=4)$; (d) Internal spatial symmetrical structure diagram of force-carrying particle $(\mathrm{n}=4)$; (e) Internal even symmetrical structure diagram of force-carrying particle $(n=4)$.

symmetrical strong interaction (as shown in Figure 1(a)) and baryon type even symmetrical strong interaction (as shown in Figure 1(b)).

1.1) The representative particle of baryon type odd symmetrical strong interaction is nucleon (including proton and neutron);

1.2) The representative particle of baryon type even symmetrical strong interaction is $\Sigma$ particles family [2] (including $\Sigma^{+}, \Sigma^{0}$ and $\Sigma^{-}$);

Similarly, meson type strong interaction can be further divided into meson type odd symmetrical strong interaction (as shown in Figure 1(c)) and meson type even symmetrical strong interaction (as shown in Figure 1(e)).

2.1) The representative particle of meson type odd symmetrical strong interaction is neutral $\mathrm{K}$ meson [3];

2.2) The representative particle of meson type odd symmetrical strong interaction is neutral $\pi$ meson [3] and charged $\mathrm{K}$ meson [3];

\subsection{Research Direction of Strong Interaction}

The five types of strong interaction have covered all strong interaction forms known in the world. This paper aims at comprehensively studying the internal symmetrical structures of hadrons and the force action from the aspect of the representative particles of the five types of strong interaction. The basic research thought is as follows:

1) Propose relevant hypothesis;

2) Infer and demonstrate according to the hypothesis;

3) Compare theoretical value and experimental value;

4) Obtain the conclusion.

The detailed analysis process includes nine steps:

1) Hypothesis for basic strong interaction; 
2) Analysis of strong interaction of nucleon (including $p$ and $n$ );

3) Analysis of strong interaction of $\Sigma$ particle family;

4) Analysis of strong interaction of $\pi$ meson;

5) Analysis of strong interaction of $K$ meson;

6) Analysis of strong interaction of atomic nucleus (e.g., deuteron and triton);

7) Analysis of strong interaction of $\Delta$ (1232);

8) Analysis of strong interaction of $\eta$ particle and $\eta^{\prime}(958)$ particle;

9) Conclusion.

\section{Hypothesis for Basic Strong Interaction}

\subsection{Internal Symmetrical Structures of Hadrons}

1) Charge type quantity of strong force: quark quantity of neutron or proton is 3 , so the charge type quantity of the corresponding strong force is 3 ; one neutron and one proton can form the simplest atomic nuclear structure, so the charge type quantity of the corresponding strong force is 2 . The corresponding charge type of the two types of the most basic strong interaction is 3 or 2 .

$$
\left\{\begin{array}{l}
\sum_{1 \leq i \leq n}^{n=3,2} e_{i}=0 \\
e_{i} \cdot e_{i}=0
\end{array}\right.
$$

where $e_{i}$ is charge type; hadrons are usually neutral and can present force action only under the distance small enough, so the sum of charge types is 0 .

It can be proved that the formula (1-1) is unsolved in the range of real number. We must extend the domain from the real to complex number, and adjust the formula to have a real root.

$$
\left\{\begin{array}{l}
\sum_{1 \leq i \leq n}^{n=3,2} e_{i}^{(*)}=0 \\
e_{i} \cdot e_{i}^{*}=0
\end{array}\right.
$$

$e_{i}$ and $e_{i}^{*}$ are conjugate complex number each other.

The following formula is set:

$$
\left\{\begin{array}{l}
e_{j}=a_{j}+b_{j} i \\
e_{j}^{*}=a_{j}-b_{j} i
\end{array}\right.
$$

$a$ and $b$ are real numbers.

Formula (1-3) are put into the formula (1-2) to obtain:

$$
\left\{\begin{array}{l}
\sum_{1 \leq i \leq n}^{n=3,2} a_{j}+b_{j} i=0 \\
\left(a_{j}+b_{j} i\right)\left(a_{j}-b_{j} i\right)=1
\end{array}\right.
$$

When $\mathrm{n}=2$,

$$
\left\{\begin{array}{l}
a_{1,2}= \pm 1 \\
b=0
\end{array}\right.
$$

A group of solutions of charge type of $\pi$ meson type strong force 


$$
e= \pm 1
$$

When $\mathrm{n}=3$, considering that neutrons and protons can combine to form stable atomic nuclei, that is to say, there must be a set of solutions (2-1),

Formulae (1-4), (2-1) are combined to solve.

$$
\left\{\begin{array}{l}
a_{1}= \pm 1, b_{1}=0 \\
a_{2}=\mp \frac{1}{2}, b_{2}=\mp \frac{\sqrt{3}}{2} i \\
a_{2}=\mp \frac{1}{2}, b_{3}=\mp \frac{\sqrt{3}}{2} i
\end{array}\right.
$$

A group of solutions of charge type of nucleon type strong force,

$$
\left\{\begin{array} { l } 
{ e _ { 1 } = \frac { 1 + \sqrt { 3 } i } { 2 } } \\
{ e _ { 2 } = \frac { 1 - \sqrt { 3 } i } { 2 } } \\
{ e _ { 3 } = - 1 }
\end{array} \quad \left\{\begin{array}{l}
e_{1}^{*}=\frac{-1-\sqrt{3} i}{2} \\
e_{2}^{*}=\frac{-1+\sqrt{3} i}{2} \\
e_{3}^{*}=1
\end{array}\right.\right.
$$

when $n=3$, the strong interaction between three forced particles can be decomposed into any two forced particles. Then we deduce the ratio of $n=3$ to $n=2$ 's Strong force intensity by formula (1-1).

$$
\frac{\sum_{j \neq m}^{n=3} e_{j} e_{m}^{*}}{2 e_{+} e_{-}}=\frac{\left(\sum_{j}^{n=3} e_{j}\right)\left(\sum_{j}^{n=3} e_{j}^{*}\right)-\sum_{j}^{n=3} e_{j} e_{j}^{*}}{\left(e_{+}+e_{-}\right)^{2}-e_{+}^{2}-e_{-}^{2}}=\frac{3}{2}
$$

2) Present two types of symmetrical structures by deuteron as shown in Figure 2.

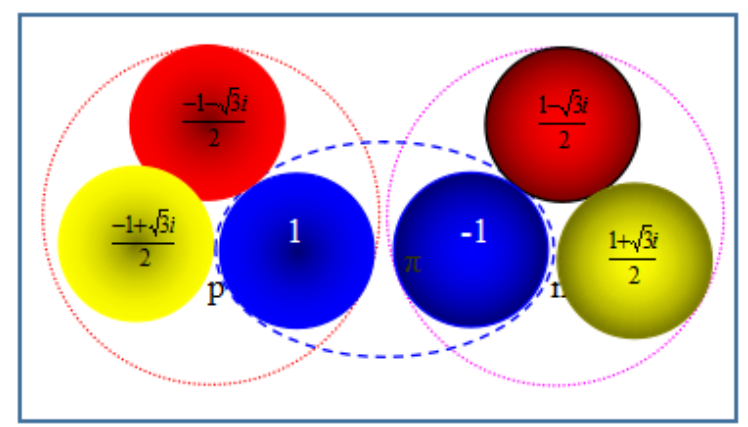

Figue 2. Two Types of Internal Symmetrical Structures of Hadrons.

\subsection{Solution of Strong Force Potential}

1) Strong force potential shall have universal attributes as the same as gravitational potential and electrostatic potential, namely:

$$
\hat{V}_{s}(r) \propto-\frac{a}{r}
$$

2) Special attributes of strong force potential: the most significant difference 
between hadrons and atoms is that hadrons only have ground state, but atoms have multiple states. In order to ensure the stability of hadrons and avoid external energy excitation, a condition for limiting state transition shall be added on the basis of Formula (1), namely:

$$
\hat{V}_{s}(r) \propto-\frac{a}{r} F(\varphi)
$$

where $\varphi$ is the ground state function of hadrons, and $F(\varphi)$ is the variable for limiting state transition.

3) a value: for the strong nuclear force as the most basic force for maintaining the internal stability of hadrons, the intensity thereof is approximate to the product of the reduced Planck constant and light velocity, and this indicates that strong nuclear force is certainly related to fluctuation. The following hypothesis is made according to such relation:

a) Strong force intensity of $\pi$ meson

$$
a=2 \times \frac{\hbar c}{2}=\hbar c
$$

b) Strong force intensity of nucleon

$$
a=3 \times \frac{\hbar c}{2}=\frac{3}{2} \hbar c
$$

4) Ground state function of hadrons: the state function of hadrons is unique and cannot be influenced by strong force potential, approximate to or as the same as that of protium atom (Schrodinger's equation is adopted to calculate the ground state function of protium atom for the most accurate comparison).

$$
\varphi(r, a)=\exp \pm r / a
$$

where $r$ and $a$ are the variables related to distance.

The state function is normalized:

$$
\varphi(r, a)=\exp \pm(r / a-1)
$$

5) $F(\varphi)$ formula

$$
F(\varphi)=\varphi^{2}=\exp \pm 2(r / a-1)
$$

6) Solution of strong force potential: two solutions of strong force potential can be obtained according to Formulae (4)-(9).

a) Strong force potential formula of $\pi$ meson

$$
\hat{V}_{S}(r)=-\frac{\hbar c}{r} \exp -2(r / a-1)
$$

or

$$
\hat{V}_{S}(r)=-\frac{\hbar c}{r} \exp +2(r / a-1)
$$

b) Strong force potential formula of nucleon

$$
\begin{aligned}
& \hat{V}_{S}(r)=-\frac{3 / 2 \hbar c}{r} \exp +2(r / a-1) \\
& \hat{V}_{S}(r)=-\frac{3 / 2 \hbar c}{r} \exp -2(r / a-1)
\end{aligned}
$$


Five issues shall be explained for 1) and 2).

1) In actual conditions, $\pi$ meson only has strong force potential formula (10);

2) The representative particle carrying (11) includes positive and negative $K$ mesons;

3) In actual conditions, nucleon only has strong force potential formula (12);

4) The representative particle carrying (12) includes $\Sigma$ particles family;

5) Strong force potential has four types, represented by Formulae (10), (11), (12) and (13).

\section{Dirac Intrinsic Equation of Nucleon}

\subsection{Description for Internal Movement of Nucleon}

Dirac equation [4] can be adopted to describe the internal movement of hadrons

$$
\left(i \gamma \frac{\partial}{\partial x^{\mu}}-m_{k}\right) u_{k}(x)=0(k=n, p, e, v)
$$

$\gamma^{\mu}$ is Dirac matrix.

Dirac eigenfunction of nucleon, established on three-quark model, is combined with positive/negative charge type Dirac function of electrons under the action of Coulomb field of protons. Three-quark model is three-body symmetrical structure and positive/negative charge is two-body symmetrical structure, so a $2 / 3$ conversion factor shall be applied between them when the three-body problem is converted into two-body problem for relevant research. The factors which represent the attributes of nucleons, such as mass and carrying force, shall be multiplied with this conversion factor, namely:

$$
\hat{H}_{i}=c \alpha \cdot p+\frac{2}{3}\left(m_{i} c^{2} \beta-\hat{V}_{e}(r)-\hat{V}_{s}(r)\right)
$$

where $i=p, n$ respectively represent proton and neutron, $V_{e}(r)$ and $V_{s}(r)$ respectively represent Coulomb field and strong interaction in nucleons.

In this way, we can establish a Hamilton operator for describing the internal state of the moving nucleons by simple mass and potential energy. When $\langle H\rangle=0$ is true, namely: nucleon energy "is developed from nothing", we can obtain the intrinsic equation of nucleons.

\subsection{Internal Symmetrical Structure of Nucleon}

Internal Odd Symmetrical Structures of Neutrons and Protons are as shown in Figure 3.

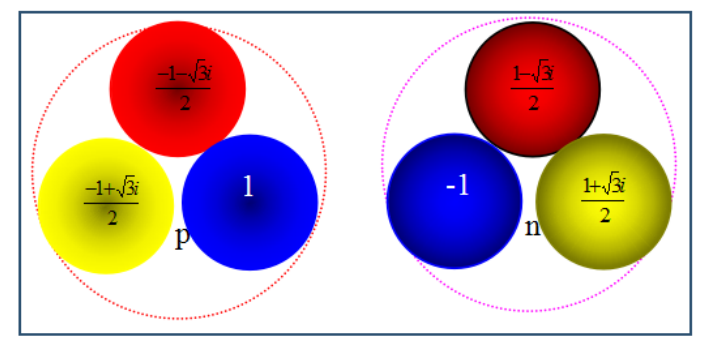

Figure 3. Internal Odd Symmetrical Structures of Neutrons and Protons. 


\subsection{Dirac Intrinsic Equation of Protons}

Firstly, we analyze protons [5]: protons carrying momentum move under strong force field and coulomb field in nucleons; then, we solve the common eigenstate function of bound states $\left(0<E<m_{p} c^{2}\right.$ or $\left.m_{n} c^{2}\right)$ and $\left(\hat{H}, \hat{K}, J^{2}, J_{z}\right)$.

Firstly, Hamilton operator of proton is as follows:

$$
\hat{H}=c \alpha \cdot p+\frac{2}{3}\left(m_{p} c^{2} \beta-3 \times\left(\frac{z_{1} z_{2} k e^{2}}{r_{1}}+\frac{z_{2} z_{3} k e^{2}}{r_{2}}+\frac{z_{1} z_{3} k e^{2}}{r_{3}}+\frac{3 / 2 \hbar c}{r} \mathrm{e}^{2\left(r / r_{0}-1\right)}\right)\right)
$$

where

$$
p=-i \hbar \nabla, \alpha=\left(\begin{array}{cc}
0 & \alpha \\
\alpha & 0
\end{array}\right), \beta=\left(\begin{array}{cc}
I & 0 \\
0 & -I
\end{array}\right)
$$

$\alpha$ and $I$ are respectively second-order pauli matrix and unit matrix, as shown below:

$$
\begin{gathered}
\alpha_{r}=\alpha \cdot \frac{\vec{r}}{r}=\alpha \cdot \frac{\vec{r}}{r}\left(\begin{array}{ll}
0 & 1 \\
1 & 0
\end{array}\right)=\alpha_{r}\left(\begin{array}{ll}
0 & I \\
I & 0
\end{array}\right) \\
\hbar \hat{K}=\beta(\Sigma \cdot l+\hbar)=(\hbar+\partial l)\left(\begin{array}{cc}
1 & 0 \\
0 & -1
\end{array}\right)
\end{gathered}
$$

where 1 is the angular momentum operator of the track, and $\hbar \Sigma / 2$ is spin angular momentum operator, namely:

$$
\sum=\alpha\left(\begin{array}{ll}
I & 0 \\
0 & I
\end{array}\right)
$$

$\frac{z_{1} z_{2}}{r_{1}}+\frac{z_{2} z_{3}}{r_{2}}+\frac{z_{1} z_{3}}{r_{3}}$ is the sum of potential energy of every two quarks of the three quarks, and coefficient 3 is the sum of the observed values of three different quarks.

Above potential energy expression includes six physical quantities, namely $z_{1}, z_{2}, z_{3}, r_{1}, r_{2}, r_{3}$, which cannot be directly solved through Dirac equation. Therefore, the potential energy expression must be simplified.

Firstly, we shall notice that all charges are integer multiples of e in coulomb field, but the basic definition is forcedly divided into three parts in quark submodel, namely:

$$
3\left(z_{1}, z_{2}, z_{3}\right)= \pm 1, \pm 2, \pm 3
$$

or

$$
z_{1}, z_{2}, z_{3}= \pm \frac{1}{3}, \pm \frac{2}{3}, \pm 1
$$

Proton charge is e, namely:

$$
z_{1}+z_{2}+z_{3}=1
$$

The following formula can be obtained through one group of values:

$$
z_{1}=-\frac{1}{3}, z_{2}=\frac{2}{3}, z_{3}=\frac{2}{3}
$$


In proton, three quarks carrying color charges are equivalent to positronelectron, and the only difference is that two symmetrical objects are changed into three objects. We can find the central distance of several quarks according to the symmetry condition in nucleon. $r_{1}, r_{2}$ and $r_{3}$ respectively represent the central distances of the three quarks, and $r$ is the radius of nucleon, as shown in Figure 4:

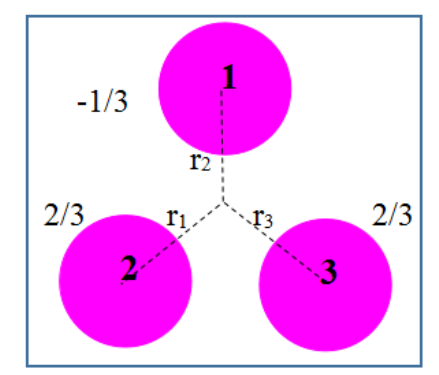

Figure 4. Symmetrical Diagram of Quarks (Coulomb Average Potential Energy =0).

$$
r_{1}=r_{2}=r_{3}=\sqrt{3} r
$$

Namely:

$$
\left\langle V_{e}(r)\right\rangle=\left\langle\frac{(-1 / 3 \times 2 / 3 \times 2+2 / 3 \times 2)}{\sqrt{3} r}\right\rangle=0
$$

Hamilton is changed as follows:

$$
\hat{H}=c \alpha \cdot p+\frac{2}{3}\left(m_{p} c^{2} \beta-\frac{3 / 2 \hbar c}{r} \mathrm{e}^{2\left(r / r_{0}-1\right)}\right)
$$

Formula (22) is converted into the following formula through the algebraic relation of $\partial, \beta, \Sigma$ :

$$
\hat{H}=c \alpha_{r} \cdot p_{r}+i \hbar c \frac{1}{r} \partial_{r} \hat{K} \beta+\frac{2}{3}\left(m_{p} c^{2} \beta-\frac{3 / 2 \hbar c}{r} \mathrm{e}^{2\left(r / r_{0}-1\right)}\right)
$$

where $p_{r}$ is radial momentum operator.

$$
\begin{gathered}
p_{r}=\frac{1}{r}(r p-i \hbar)=i \hbar\left(\frac{1}{r}+\frac{\partial}{\partial r}\right) \\
J=l+\frac{\hbar}{2} \sum
\end{gathered}
$$

$\hat{H}, \hat{K}, J^{2}, J_{z}$ can be proven to be mutually commuted, and the eigenvalues thereof are as follows:

$$
\begin{aligned}
& J^{2}=j(j+1) \hbar^{2}, j=\frac{1}{2}, \frac{3}{2}, \cdots \\
& J_{z}=m j \hbar, m j=j, j-1, \cdots \\
& \hat{K}=K= \pm\left(j+\frac{1}{2}\right)= \pm 1, \pm 2, \cdots
\end{aligned}
$$

As a result, common eigenfunction of $\left(\hat{H}, \hat{K}, J^{2}, J_{z}\right)$ can be divided into two types: 
1) $K=j+\frac{1}{2}$

$$
\varphi=\left(\begin{array}{l}
\varphi_{1} \\
\varphi_{2} \\
\varphi_{3} \\
\varphi_{4}
\end{array}\right)=\left(\begin{array}{l}
\phi_{j m j}^{A} f(r) \\
\phi_{j m j}^{B} g(r)
\end{array}\right)=\left(\begin{array}{c}
\phi_{j m j}^{A} \frac{F(r)}{r} \\
i \phi_{j m j}^{B} \frac{G(r)}{r}
\end{array}\right)
$$

2) $K=-\left(j+\frac{1}{2}\right)$

$$
\varphi=\left(\begin{array}{l}
\varphi_{1} \\
\varphi_{2} \\
\varphi_{3} \\
\varphi_{4}
\end{array}\right)=\left(\begin{array}{l}
\phi_{j m j}^{B} f(r) \\
\phi_{j m j}^{A} g(r)
\end{array}\right)=\left(\begin{array}{c}
\phi_{j m j}^{B} \frac{F(r)}{r} \\
i \phi_{j m j}^{A} \frac{G(r)}{r}
\end{array}\right)
$$

and $f(r)=\frac{F(r)}{r}, g(r)=\frac{G(r)}{r}$

For convenient calculation, $\phi_{j m j}^{A}$ and $\phi_{j m j}^{B} g(r)$ are set as two-component $\left(J^{2}, J_{z}\right)$ eigenfunction $(\theta, \varphi$ function, irrelevant to $r)$.

Formula (28) is put into intrinsic equation of energy.

$$
H \varphi=E \varphi
$$

The following formula is obtained

$$
p_{r} \frac{F(r)}{r}=-i \hbar \frac{1}{r} \frac{\mathrm{d} F}{\mathrm{~d} r}
$$

to establish $F(r), G(r)$ simultaneous equations:

$$
\left\{\begin{array}{l}
\frac{\mathrm{d} F}{\mathrm{~d} r}-\frac{K}{r} F=\left(\frac{\frac{2}{3} m c^{2}+E}{\hbar c}+\frac{1}{r} \mathrm{e}^{-2\left(r / r_{0}-1\right)}\right) G \\
\frac{\mathrm{d} G}{\mathrm{~d} r}+\frac{K}{r} G=\left(\frac{\frac{2}{3} m c^{2}-E}{\hbar c}-\frac{1}{r} \mathrm{e}^{-2\left(r / r_{0}-1\right)}\right) F
\end{array}\right.
$$

where $\alpha=e^{2} / \hbar c$ is fine structure constant.

In following paragraph, $F$ and $G$ actions are researched around singular point $r=0, \infty$.

When $r \rightarrow \infty$ is true, Formula (30) can be approximately valued as:

$$
\frac{\mathrm{d} F}{\mathrm{~d} r} \approx \frac{\frac{2}{3} m_{p} c^{2}+E^{2}}{\hbar c} G, \frac{\mathrm{d} G}{\mathrm{~d} r} \approx \frac{\frac{2}{3} m_{p} c^{2}-E^{2}}{\hbar c} F
$$

Therefore,

$$
\frac{\mathrm{d} F}{\mathrm{~d} r} \approx \frac{\frac{4}{9} m_{p} c^{2}-E^{2}}{\hbar^{2} c^{2}} F, \frac{\mathrm{d} G}{\mathrm{~d} r} \approx \frac{\frac{4}{9} m_{p} c^{2}-E^{2}}{\hbar^{2} c^{2}} G
$$

Obviously, physically allowable progressive action of $G$ and $F$ is as follows: 


$$
r \rightarrow \infty, F, G \sim \mathrm{e}^{-\frac{\sqrt{\frac{2}{3} m_{p} c^{2}-E^{2}}}{\hbar c} r}
$$

When $r \rightarrow 0$ is true, $F$ and $G$ shall be at the size of the same level according to Formula (31).

$$
F \rightarrow C_{1} r^{s}, G \sim C_{2} r^{s}
$$

Above formula is put into Formula (30) to obtain:

$$
\left\{\begin{array}{l}
(s-K) C_{1}-\mathrm{e}^{2\left(r / r_{0}-1\right)} C_{2}=0 \\
(s+K) C_{2}+\mathrm{e}^{2\left(r / r_{0}-1\right)} C_{1}=0
\end{array}\right.
$$

$C_{1}$ and $C_{2}$ cannot be set as 0 at the same time, so the following condition must be met.

$$
\left|\begin{array}{cc}
(s-K) & -\mathrm{e}^{2\left(r / r_{0}-1\right)} \\
\mathrm{e}^{2\left(r / r_{0}-1\right)} & (s+K)
\end{array}\right|=0
$$

Accordingly, the following solution is obtained:

$$
s= \pm \sqrt{K^{2}-\left(\mathrm{e}^{2\left(r / r_{0}-1\right)}\right)^{2}}
$$

When applying the global approach to find the movement of nucleon in its own field, it is necessary to notice that its static mass is not changed. Namely, when $\langle H\rangle=0$ or $s=0$ is true, we can obtain a nucleon only influenced by its internal action, thus avoiding the influence of external momentum.

$$
\left(\mathrm{e}^{2\left(r / r_{0}-1\right)}\right)^{2}=K^{2}
$$

In above formula, the left part is a continuous value changed along with $r$, and the right part is discontinuous value which can be only positive and negative integers. Obviously, the two parts are unequal to each other, and the unique feasible method is to set the following equation:

$$
\mathrm{e}^{2\left(r / r_{0}-1\right)}=\text { constant }
$$

Namely:

$$
r=r_{0}
$$

Proton only has one state, namely its ground state. When $K^{2}=1$ is true, the following definition is established according to the relation between mass and fluctuation:

$$
r_{0}=\frac{\frac{3}{2} \hbar}{m_{p} c}
$$

The state equation of proton can be solved as follows:

When $K=1$ is true,

$$
F=-G=\mathrm{e}^{-r / r_{0}}
$$

Above formula can be met. 
When $K=-1$ is true,

$$
F=-G=\mathrm{e}^{r / r_{0}}
$$

Function convergence requirement cannot be met.

\subsection{Dirac Intrinsic Equation of Neutron}

Neutron has two states around decay, namely: neutron state similar to proton state, and proton-electron state after fission, so there are two Hamilton operators.

$$
\left\{\begin{array}{l}
\hat{H}_{1}=\left[c \partial \cdot p_{1}+\frac{2}{3}\left(m_{n} c^{2} \beta-\left(3\left(\frac{z_{1} z_{2} k e^{2}}{r_{1}}+\frac{z_{2} z_{3} k e^{2}}{r_{2}}+\frac{z_{1} z_{3} k e^{2}}{r_{3}}\right)+\frac{3 / 2 \hbar c}{r} \mathrm{e}^{2\left(r / r_{0}-1\right)}\right)\right)\right] p_{1} \\
\hat{H}_{2}=\left(c \partial \cdot p+m_{e} c^{2}-\frac{e^{2}}{r}\right) p_{2}
\end{array}\right.
$$

$p_{1}$ and $p_{2}$ respectively represent the probabilities of the two states, so $p_{1}+p_{2}=1$ is true.

Similarly to Formula (24), above formula can be simplified as follows:

$$
\left\{\begin{array}{l}
\hat{H}_{1}=\left[c \partial_{r} \cdot p_{r}+i \hbar c \frac{1}{r} \alpha_{r} \hat{K} \beta+\frac{2}{3}\left(m_{p} c^{2} \beta-\left(\frac{1}{\sqrt{3}} \frac{k e^{2}}{r}+\frac{3 / 2 \hbar c}{r} \mathrm{e}^{2\left(r / r_{0}-1\right)}\right)\right)\right] p_{1} \\
\hat{H}_{2}=\left(c \alpha \cdot p+m_{e} c^{2}-\frac{e^{2}}{r}\right) p_{2}
\end{array}\right.
$$

Three quarks are symmetrical with each other and meet the mutual mass center as shown in Formula (21), the symmetrical diagram of quarks as shown in Figure 5.

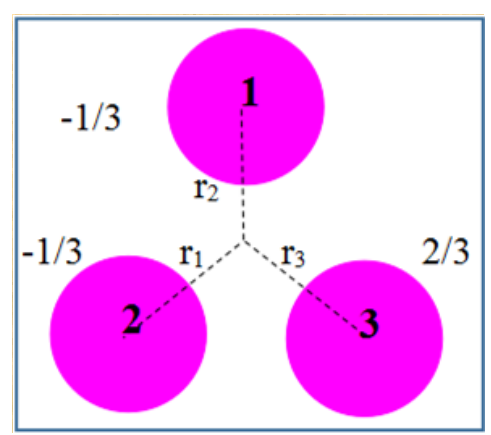

Figure 5. Symmetrical Diagram of Quarks.

Namely:

$$
\left\langle V_{e}(r)\right\rangle=\left\langle 3 \times \frac{(-1 / 3 \times 2 / 3 \times 2+1 / 3 \times 2)}{\sqrt{3} r} e^{2}\right\rangle=-\frac{e^{2}}{\sqrt{3} r}
$$

We find $p_{1}$ and $p_{2}$ as follows:

For converting Formula (39) into the fine structure form similarly with Formula (25) for solution, we firstly need to change $s$. 
The following formula is defined:

$$
s^{2}=s_{1}^{2}+s_{2}^{2}
$$

The following formula can be obtained:

$$
\left\{\begin{array}{l}
s_{1}^{2}=\left(K_{1}^{2}-\left(\frac{2 \sqrt{3}}{9} \alpha+\mathrm{e}^{2\left(r_{n} / r_{0}-1\right)}\right)^{2}\right) p_{1}^{2} \\
s_{2}^{2}=\left(K_{2}^{2}-\alpha^{2}\right) p_{2}^{2}
\end{array}\right.
$$

Now, we need to find a probability in decay intermediate process to determine $p_{1}$ and $p_{2}$.

There are two charges as $2 / 3$ u quarks in neutron, and each charge may decay into one negative particle. Due to the repulsive interaction between the two particles, the following two groups of potential energy can be found.

Potential energy including coulomb field or potential energy including strong force field and coulomb field (d Quark Decays into u Quark and Probably Releases Two Negative Charges Relevant or Irrelevant to Each Other as shown in Figure 6).

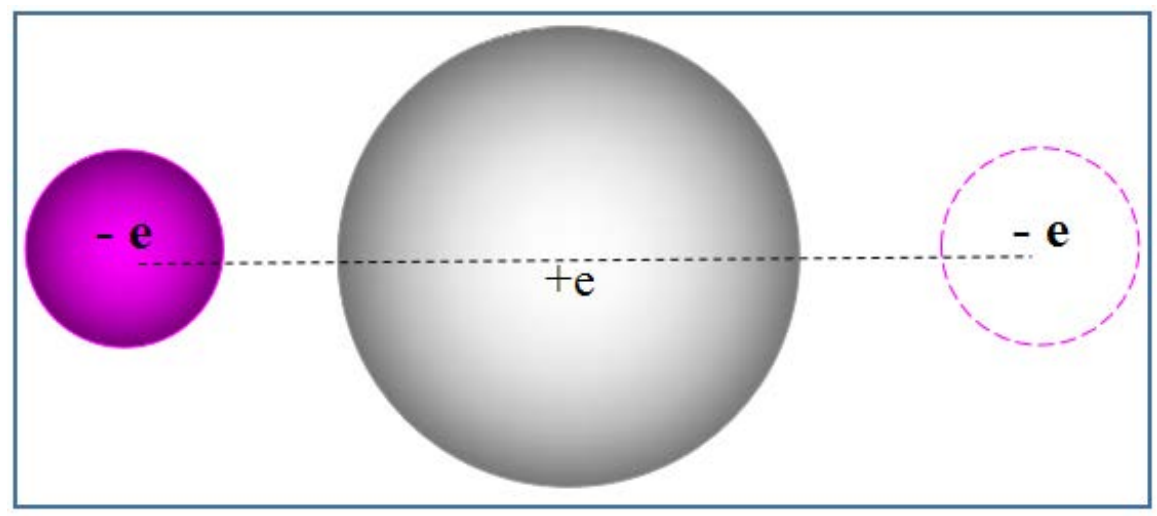

Figure 6. d Quark Decays into u Quark and Probably Releases Two Negative Charges Relevant or Irrelevant to Each Other.

$$
\left\{\begin{array}{l}
V(r)_{e}=2 \times \frac{k e^{2}}{r}-\frac{1}{2} \frac{k e^{2}}{r}=\frac{3}{2} \frac{k e^{2}}{r}=\frac{3}{2} \alpha \frac{\hbar c}{r} \\
V(r)_{n}=\frac{\hbar c}{r}+\frac{1}{2} \frac{k e^{2}}{r}=\frac{\hbar c(1+1 / 2 \alpha)}{r}
\end{array}\right.
$$

Probability is directly proportional to the square of potential energy, namely: the following formula can be obtained according to above formula:

$$
\left\{\begin{array}{c}
p_{1}=\frac{1+\frac{1}{2} \alpha}{1+2 \alpha} \\
p_{2}=\frac{\frac{3}{2} \alpha}{1+2 \alpha}
\end{array}\right.
$$

Formulae (41)-(43) are combined to obtain: 


$$
s^{2}=\left(K_{1}^{2}-\left(\frac{2 \sqrt{3}}{9} \alpha+\mathrm{e}^{2\left(r_{n} / r_{0}-1\right)}\right)^{2}\right)\left(\frac{1+\frac{1}{2} \alpha}{1+2 \alpha}\right)^{2}+\left(K_{2}^{2}-\alpha^{2}\right)\left(\frac{\frac{3}{2} \alpha}{1+2 \alpha}\right)^{2}
$$

When $\langle H\rangle=0$ or $s=0$ is true, we obtain a neutron only influenced by its internal action, thus avoiding the influence of external kinetic energy.

$$
0=\left(K_{1}^{2}-\left(\frac{2 \sqrt{3}}{9} \alpha+\mathrm{e}^{2\left(r_{n} / r_{0}-1\right)}\right)^{2}\right)\left(\frac{1+\frac{1}{2} \alpha}{1+2 \alpha}\right)^{2}+\left(K_{2}^{2}-\alpha^{2}\right)\left(\frac{\frac{3}{2} \alpha}{1+2 \alpha}\right)^{2}
$$

Similarly with Formula (35), the following formula can be defined:

$$
r_{0}=\frac{\frac{3}{2} \hbar}{m_{n} c}
$$

Formula (45) is converted into:

$$
0=\left(K_{1}^{2}-\left(\frac{2 \sqrt{3}}{9} \alpha+\mathrm{e}^{2\left(m_{p} / m_{n}-1\right)}\right)^{2}\right)\left(\frac{1+\frac{1}{2} \alpha}{1+2 \alpha}\right)^{2}+\left(K_{2}^{2}-\alpha^{2}\right)\left(\frac{\frac{3}{2} \alpha}{1+2 \alpha}\right)^{2}
$$

At the moment, neutron is under ground state, namely:

$$
K_{1}^{2}=K_{2}^{2}=1
$$

Accordingly, proton-neutron mass ratio is obtained as follows:

$$
\frac{m_{p}}{m_{n}}=1+\frac{1}{2} \ln \left(\sqrt{1+\frac{9}{4} \alpha^{2}\left(1-\alpha^{2}\right) \div\left(1+\frac{1}{2} \alpha\right)^{2}}-\frac{2 \sqrt{3}}{9} \alpha\right)
$$

The influence of proton recoil and weak decay on $\alpha^{2}$ is not considered in Formula (47). Therefore, we will continuously discuss relevant problem.

1) Influence of proton recoil on $\alpha^{2}$ :

Diagram of Recoil Movement between Proton and Electron is as shown in Figure 7.

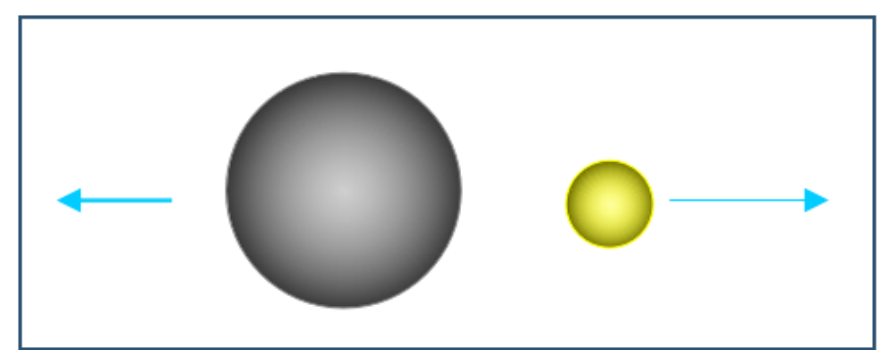

Figure 7. Diagram of Recoil Movement between Proton and Electron.

Namely:

$$
\left\{\begin{array}{l}
m_{p} v_{p}=m_{e} v_{e} \\
m_{p} v_{p} r_{e}=m_{e} v_{e} r_{e}=\hbar
\end{array}\right.
$$




$$
E=\frac{1}{2} \times m_{e} c^{2} \times \frac{9}{4} \alpha^{2}\left(1+\frac{m_{e}}{m_{p}}\right)
$$

Namely, the influence of proton recoil on $\alpha^{2}$ is as follows:

$$
\frac{9}{4} \alpha^{2}\left(1+\frac{m_{e}}{m_{p}}\right)
$$

2) Influence of weak decay on $\alpha^{2}$ :

$\mathrm{z}$-axis is taken as the observation axis; in consideration of the similarity between weak force and spinning interaction, the weak force formula is set as follows:

$$
\begin{aligned}
& \hat{V}(r)_{w}=-\frac{8 \pi}{3} e_{e} e_{v e} \sigma(r) \\
& \left\{\begin{array}{l}
e_{e}=-\frac{e}{m_{e} c} S_{e} \cdot \frac{1}{2}\left(\alpha_{x}-i \alpha_{y}\right) \\
e_{v_{e}}=-\frac{e}{m_{e} c} S_{v_{e}} \cdot \frac{1}{2}\left(\alpha_{x}+i \alpha_{y}\right)
\end{array}\right.
\end{aligned}
$$

The calculation method for weak potential is as the same as that for the superfine action between proton and electron under s state of hydrogen atom $(1=0)$.

$$
S_{e} \cdot S_{v_{e}}=\frac{1}{4} \hbar^{2}
$$

Finally, the energy for weak force decay is calculated as follows:

$$
E^{(1)}=-\frac{1}{3} m_{e} c^{2} \alpha^{4}
$$

In consideration of the influence of proton recoil, the corrected energy is as follows:

$$
E^{(1)}=-\frac{1}{3} m_{e} c^{2} \alpha^{4}\left(1+\frac{m_{e}}{m_{p}}\right)
$$

The influence on $\alpha^{2}$ is as follows:

$$
2 \times \frac{1}{3} \alpha^{2}\left(1+\frac{m_{e}}{m_{p}}\right)
$$

The correct proton-neutron mass ratio is as follows:

$$
\frac{m_{p}}{m_{n}}=1+\frac{1}{2} \ln \left(\sqrt{1+\frac{9}{4} \alpha^{2}\left(1-\alpha^{2}\right) \div\left(1+\frac{1}{2} \alpha\right)^{2}\left(1+\frac{m_{e}}{m_{p}}\right)\left(1-\frac{2}{3} \alpha^{2}\right)}-\frac{2 \sqrt{3}}{9} \alpha\right)
$$

The experimental value of fine structure constant is set as $\alpha=0.0072973525664$ to obtain the theoretical value of Formula (52) as 0.99862347888 . The experimental value of this constant can be checked in NIST [1] official website as $\left(\frac{m_{p}}{m_{n}}\right)_{\exp }=0.99862347844(51)$. We can find that the theoretical value has a spectacular accuracy, thus indicating that it is feasible for 
us to describe the internal movement state of nucleon by Dirac equation. Therefore, this can be taken as the necessary evidence for us to verify the strong nuclear force potential formula.

When neutron is under the ground state $s=0$, the state equation of neutron, solved thereby, can meet Formula (36).

\subsection{Internal Strong Interaction of $\Sigma$ Baryon}

\subsubsection{Internal Symmetrical Structure of $\Sigma$ Baryon}

$\Sigma$ particles family includes $\Sigma^{+}, \Sigma^{0}$ and $\Sigma^{-}$, respectively corresponding to the quark compositions (uus), (dus) and (dds). s quark is not a stable quark, and decays into a stable structure according to the form of $\mathrm{s} \rightarrow \mathrm{K}^{0}\left(\mathrm{~K}^{ \pm}\right)+\mathrm{d}(\mathrm{u})$, as shown in Figure 8.

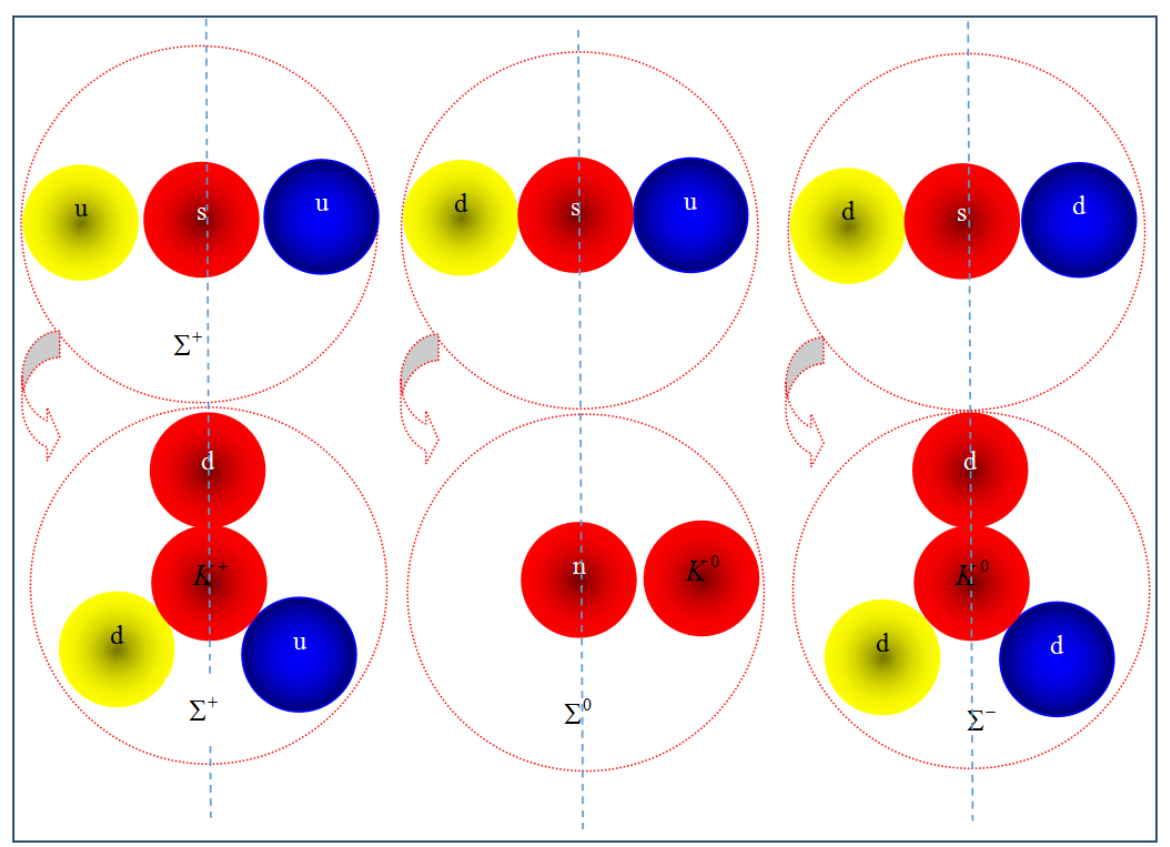

Figure 8. Internal Symmetrical Structure of $\Sigma$ baryon.

\subsubsection{Dirac Function of $\Sigma$ Baryon}

Hamilton operator of $\Sigma$ baryon is as follows:

$$
\begin{gathered}
\hat{H}_{\Sigma+}=\left[c \partial_{r} \cdot p_{r}+i \hbar c \frac{1}{r} \alpha_{r} \hat{K} \beta+\frac{2}{3}\left(m_{\Sigma+} c^{2} \beta-\left(5 \times \frac{1}{3 \sqrt{3}} \frac{k e^{2}}{r}+\frac{3 / 2 \hbar c}{r} \mathrm{e}^{-2\left(r / r_{0}-1\right)}\right)\right)\right] \\
\hat{H}_{\Sigma 0}=\left[c \partial_{r} \cdot p_{r}+i \hbar c \frac{1}{r} \alpha_{r} \hat{K} \beta+\frac{2}{3}\left(m_{\Sigma 0} c^{2} \beta-\left(\frac{3 / 2 \hbar c}{r} \mathrm{e}^{-2\left(r / r_{0}-1\right)}\right)\right)\right] \\
\hat{H}_{\Sigma-}=\left[c \partial_{r} \cdot p_{r}+i \hbar c \frac{1}{r} \alpha_{r} \hat{K} \beta+\frac{2}{3}\left(m_{\Sigma-} c^{2} \beta-\left(7 \times \frac{1}{3 \sqrt{3}} \frac{k e^{2}}{r}+\frac{3 / 2 \hbar c}{r} \mathrm{e}^{-2\left(r / r_{0}-1\right)}\right)\right)\right]
\end{gathered}
$$

The process for solving Dirac equation of $\Sigma$ baryon is consistent with the process for solving Dirac equation of proton. The state function finally obtained can meet Formula (36). 
The mass ratio of the three particles of $\Sigma$ particles family can be obtained as follows:

$$
\begin{aligned}
m_{\Sigma^{+}}: m_{\Sigma^{0}}: m_{\Sigma^{-}} & =\left(1-0.5 \ln \left(1-\frac{10 \sqrt{3}}{27} \alpha\right)\right)^{-}: 1:\left(1-0.5 \ln \left(1+\frac{14 \sqrt{3}}{27} \alpha\right)\right)^{-} \\
& =0.997659: 1: 1.00328
\end{aligned}
$$

The experimental value of mass ratio is put to obtain:

$$
m_{\Sigma^{+} \exp }: m_{\exp }: m_{\text {exp }}=0.997257: 1: 1.00403
$$

The experimental value is basically consistent with the theoretical value.

\subsubsection{Estimation of Mass of $\Sigma$ Baryon}

$m_{\Sigma^{0}}=m_{n}+1 / 2 m_{K^{0}}$ is assumed to estimate the mass of three $\Sigma$ baryon particles, and the estimated value is compared with the experimental value (as shown in Table 1).

Table 1. Three $\Sigma$ baryon's mass comparison table.

\begin{tabular}{lcccc}
\hline $\begin{array}{c}\text { Particle } \\
\text { Name }\end{array}$ & Mass Formula & $\begin{array}{c}\text { Theoretical } \\
\text { Value } A \text { of } \\
\text { Mass }(\mathrm{Mev})\end{array}$ & $\begin{array}{c}\text { Experimental } \\
\text { Value }[2] B \text { of } \\
\text { Mass }(\mathrm{Mev})\end{array}$ & $\begin{array}{c}\text { Error Rate }= \\
(A / B-1)^{*} \%\end{array}$ \\
\hline$\Sigma^{+}$ & $m_{\Sigma^{+}}=m_{\Sigma^{0}}\left(1-0.5 \ln \left(1-\frac{10 \sqrt{3}}{27} \alpha\right)\right)^{-}$ & 1185.60 & $1189.37 \pm 0.07$ & $-0.32 \%$ \\
$\Sigma^{0}$ & $m_{\Sigma^{0}}$ & 1188.38 & $1192.642 \pm 0.024$ & $-0.35 \%$ \\
$\Sigma^{-}$ & & & & $-0.43 \%$ \\
\hline
\end{tabular}

Small error exists between theoretical value and experimental value.

\section{Dirac Intrinsic Equation of $\pi$ Meson}

\subsection{Internal Symmetrical Structure of $\pi$ Meson}

Internal Even Symmetrical Structure of $\pi$ Meson is as shown in Figure 9.

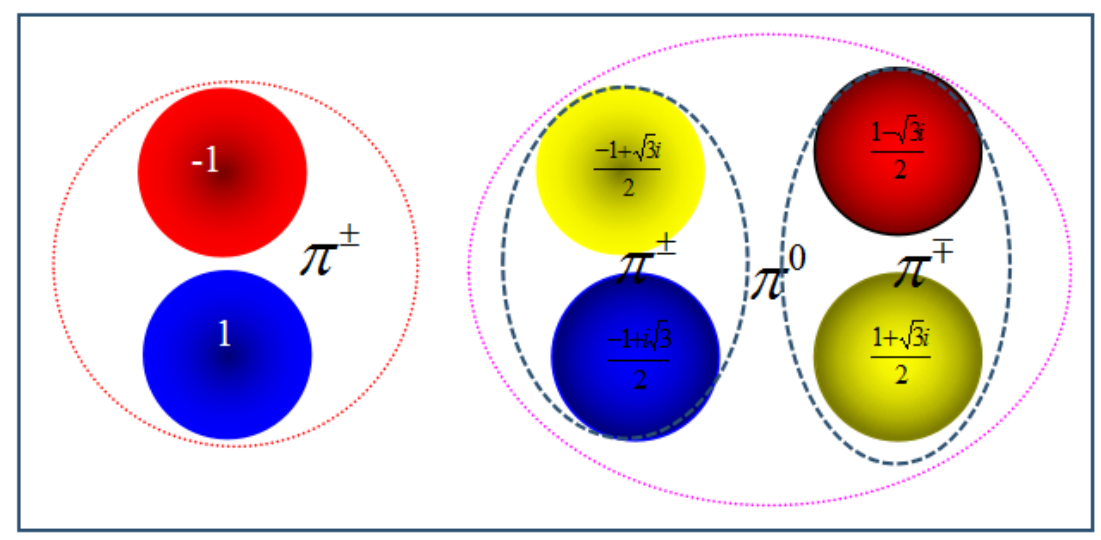

Figure 9. Internal Even Symmetrical Structure of $\pi$ Meson. 


\subsection{Dirac Intrinsic Equation of $\pi_{ \pm}$}

We firstly analyze $\pi_{ \pm}$, and this is simple for us to understand, because we have solved Dirac intrinsic equations of proton and neutron.

Hamilton operator of $\pi_{ \pm}$is as follows:

$$
\hat{H}=c \alpha \cdot p+\left(m_{\pi^{ \pm}} c^{2} \beta-\left(-\frac{k e^{2}}{r}+\frac{\hbar c}{r} \mathrm{e}^{2\left(r / a_{0}-1\right)}\right)\right)
$$

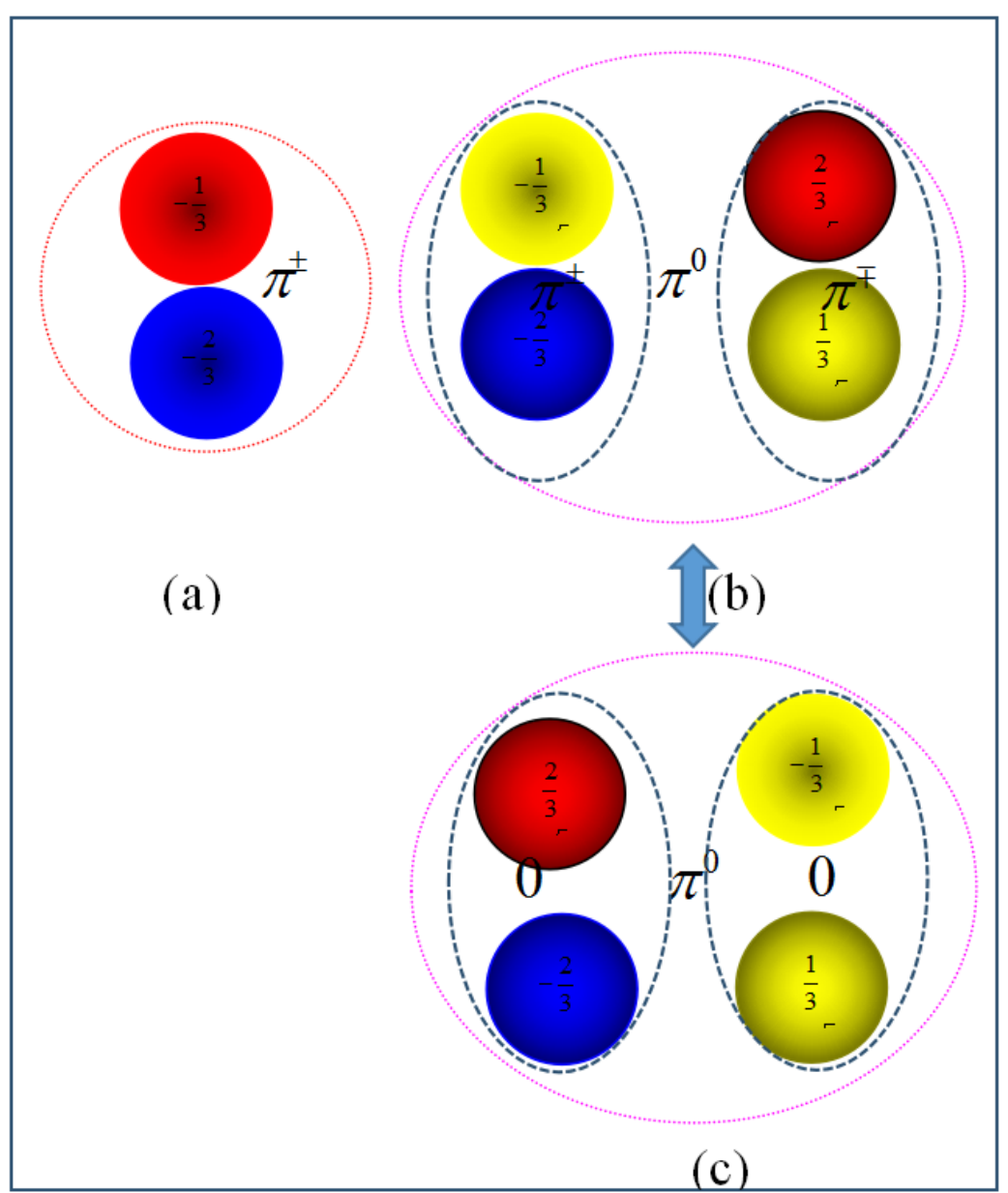

Figure 10. (a) Internal Charge Symmetry of Positive-Negative $\pi$ Meson; (b) Internal Charge Symmetry of Zero $\pi$ Meson; (c) Charge Symmetry in Zero $\pi$ Meson under Violation of Minimum Charge Value.

Notably, two quarks in meson have no electrostatic force action, but under decay state, electron and anti-electron neutrino form a special electrostatic interaction of the same charge, which seems as an electrostatic field between two electrons. Anti-electron neutrino like a mirror projects an electron with the attribute as the same as that of primary electron, and then two electrons have electromagnetic interaction (as shown in Figure 10).

Namely:

$$
p=-i \hbar \nabla, \alpha=\left(\begin{array}{cc}
0 & \alpha \\
\alpha & 0
\end{array}\right), \beta=\left(\begin{array}{cc}
I & 0 \\
0 & -I
\end{array}\right)
$$


When $\langle H\rangle=0$ is true, namely: when we obtain a static $\pi_{ \pm}$, a group of necessary conditions can be obtained as follows:

$$
\left\{\begin{array}{l}
K^{2}=1 \\
a_{0}=\frac{\hbar}{m_{\pi^{ \pm}} c}
\end{array}\right.
$$

When $K=1$ is true, $F=-G=\mathrm{e}^{-r / r_{ \pm}}$can meet the requirement.

When $K=-1$ is true, $F=-G=\mathrm{e}^{-r / r_{ \pm}}$cannot meet function convergence requirement.

\subsection{Dirac Intrinsic Equation of $\pi^{0}$}

Hamilton operator of $\pi^{0}$ is as follows:

$$
\hat{H}=c \alpha \cdot p+\left(m_{\pi_{ \pm}} c^{2} \beta-\left(2 \times 4 \times \frac{k e^{2}}{2 r}+\frac{\hbar c}{2 r} \mathrm{e}^{-2\left(r / a_{0}-1\right)}\right)\right)
$$

Namely:

$$
p=-i \hbar \nabla, \alpha=\left(\begin{array}{cc}
0 & \alpha \\
\alpha & 0
\end{array}\right), \beta=\left(\begin{array}{cc}
I & 0 \\
0 & -I
\end{array}\right)
$$

When $\langle H\rangle=0$ is true, namely: when we obtain a static $\pi_{ \pm}$, a group of necessary conditions can be obtained as follows:

$$
\left\{\begin{array}{l}
K^{2}=1 \\
a_{0}=\frac{\hbar}{m_{\pi^{ \pm}} c}
\end{array}\right.
$$

At the moment,

$$
\frac{m_{\pi_{ \pm}}}{m_{\pi_{0}}}=\frac{1-\frac{1}{2} \ln (1-8 \alpha)}{1-\frac{1}{2} \ln (1+\alpha)}
$$

When $K=1$ is true, $F=-G=\mathrm{e}^{-r / r_{ \pm}}$can meet the requirement.

When $K=-1$ is true, $F=-G=\mathrm{e}^{-r / r_{ \pm}}$cannot meet function convergence requirement.

The condition shown in Figure 10(c) is not considered in above formula, and the specific value of such condition and common symmetry condition is exactly the specific value of strong force and electrostatic force, namely: $1: 8 \alpha$, so the above formula is corrected as follows:

$$
\frac{m_{\pi_{ \pm}}}{m_{\pi_{0}}}=\frac{1-\frac{1}{2} \ln \left(1-8 \alpha-80 \alpha^{2} / 9\right)}{1-\frac{1}{2} \ln (1+\alpha)}=1.034087
$$

The experimental value of the mass ratio of charged $\pi$ meson and neutral $\pi$ meson cannot be found in references, so the experimental value of the mass ratio of the two $\mathrm{K}$ mesons is obtained through the mass [3] division:

$$
\frac{m_{\pi_{ \pm} \mathrm{exp}}}{m_{\pi_{0} \exp }}=\frac{139.57018 \pm 0.00035}{134.9766 \pm 0.0006}=1.034032 \pm \ldots
$$


Formula (59) is divided by Formula (60) to obtain:

$$
=\frac{1.034087}{1.034032} \times 100 \%-1=5.3 \times 10^{-5}
$$

Error rate is 0.0000053 , thus indicating the theoretical value is consistent with the experimental value.

\section{Dirac Intrinsic Equation of K Meson}

\subsection{Internal Symmetrical Structure of K Meson [6]}

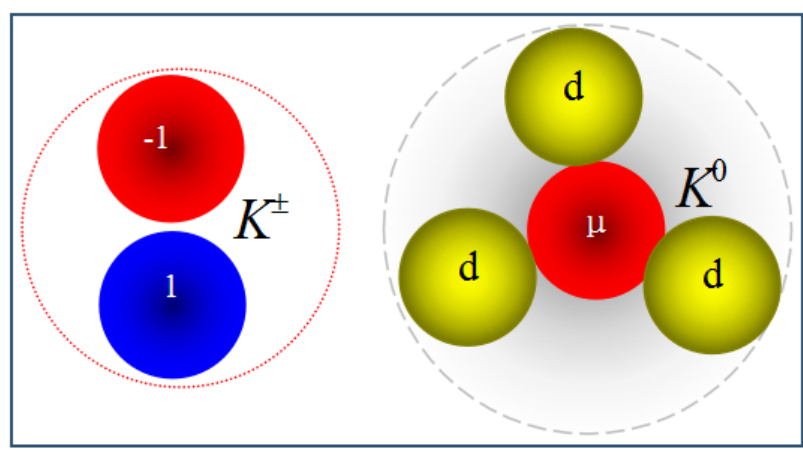

Figure 11. Internal Even Symmetrical Structure of K Meson.

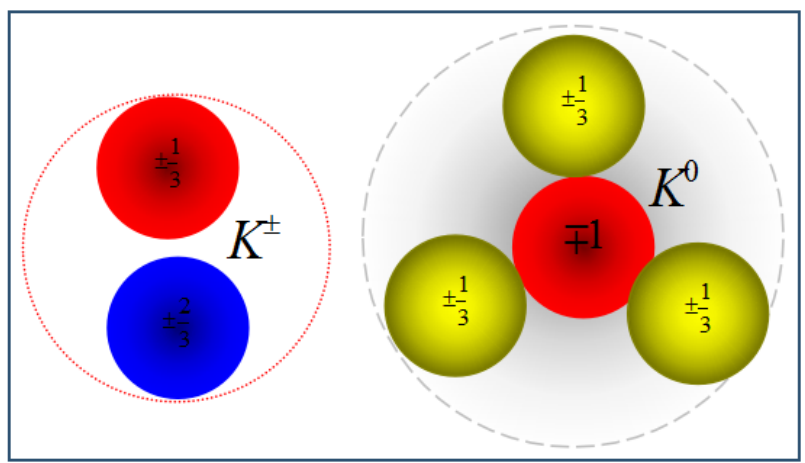

Figure 12. Internal Charge Symmetry of K Meson.

\subsection{Dirac Intrinsic Equation of K Meson}

The process for solving Dirac equation of $\mathrm{K}$ meson is similar with the process for solving the same of $\pi$ meson. But such process is independently described in following paragraph for three purposes: 1) wide applicable scope of the paper: except $\mathrm{K}$ meson which also includes s quark, other particles in this paper are composed of two quarks-u and d quarks, so the introduction to such process can further widen the applicable scope of strong interaction verified in this paper; 2) the internal symmetrical type of charged $\mathrm{K}$ meson is odd symmetry, and such structure rarely exists among mesons; 3 ) the strong force potential of charged $\mathrm{K}$ meson is as shown in Formula (11), thus proving the existence of such strong force potential (as shown in Figure 11, Figure 12).

Finally, the formula for the mass ratio of charged $\mathrm{K}$ meson and neutral $\mathrm{K}$ meson is as follows: 


$$
\frac{m_{K_{ \pm}}}{m_{K_{0}}}=\frac{1+0.5 \ln (1-4(1-1 / 3 \sqrt{3}) \alpha)}{1-0.5 \ln (1+\alpha)}=0.991678
$$

The experimental value of the mass ratio of charged $\mathrm{K}$ meson and neutral $\mathrm{K}$ meson cannot be found in references, so the experimental value of the mass ratio of the two $\mathrm{K}$ mesons is obtained through the mass [3] division:

$$
\frac{m_{K_{ \pm} \exp }}{m_{K_{0} \exp }}=\frac{493.677 \pm 0.016}{497.648 \pm 0.024}=0.992020 \pm \cdots
$$

Formula (70) is divided by Formula (71) to obtain:

$$
=\frac{0.991678}{0.992020} \times 100 \%-1=3.4 \times 10^{-4}
$$

Error rate is 0.000034 , thus indicating the theoretical value is consistent with the experimental value.

\section{Strong Interaction of Atomic Nucleus}

Internal strong force action of atomic nucleus is sourced from $\pi$ meson exchange between proton and neutron. The reaction formula is expressed as follows:

$$
\mathrm{n} \Leftrightarrow \mathrm{p}+\pi^{ \pm}
$$

The internal strong interaction of deuteron is generated by $\pi$ meson exchange between single proton and single neutron through replacement reaction as shown in Formula (73). Notably, $\pi$ meson exchange also exists between two neutrons, but the replacement between two neutrons cannot be distinguished, so there is no force effect or force action (as shown in Figure 13).

The internal strong interaction of deuteron is the most basic internal strong interaction of atomic nucleus. The four most representative and simplest particlesdeuteron, triton, He-3 and He-4 will be researched in following paragraph to explain the force action in atomic nucleus (as shown in Figure 13).

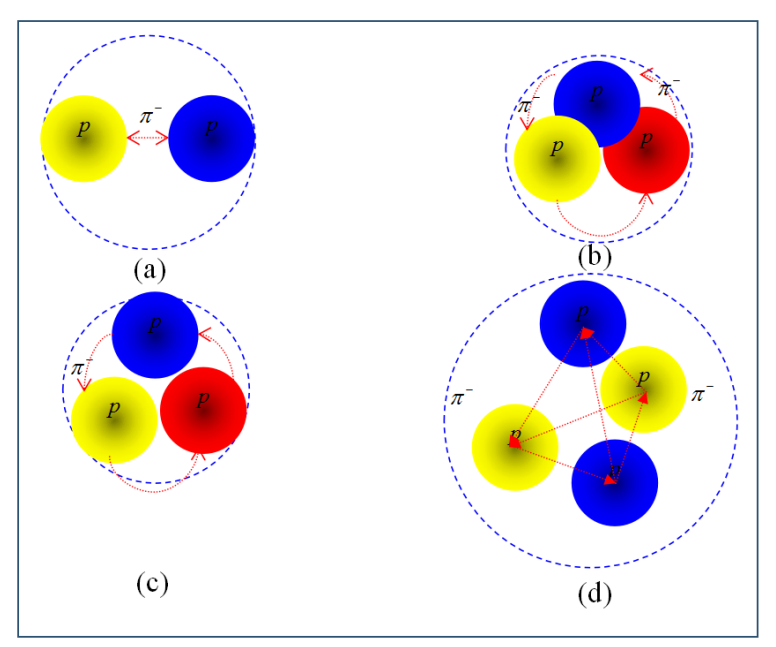

Figure 13. (a) $\pi$ Meson Loop-exchange State in Deuteron; (b) $\pi$ Meson Loop-exchange State in Triton; (c) $\pi$ Meson Loop-exchange State in He-3; (d) $\pi$ Meson Loop-exchange State in He-4. 


\subsection{Strong Interaction of Deuteron}

Classical theory is adopted to solve the combined potential of deuteron. Deuteron is an atomic nucleus formed by the combination of one proton and one neutron under strong action, and the internal structure of deuteron can meet angular momentum conservation and stress balance (as shown in Figure 13(a) and Figure 14).

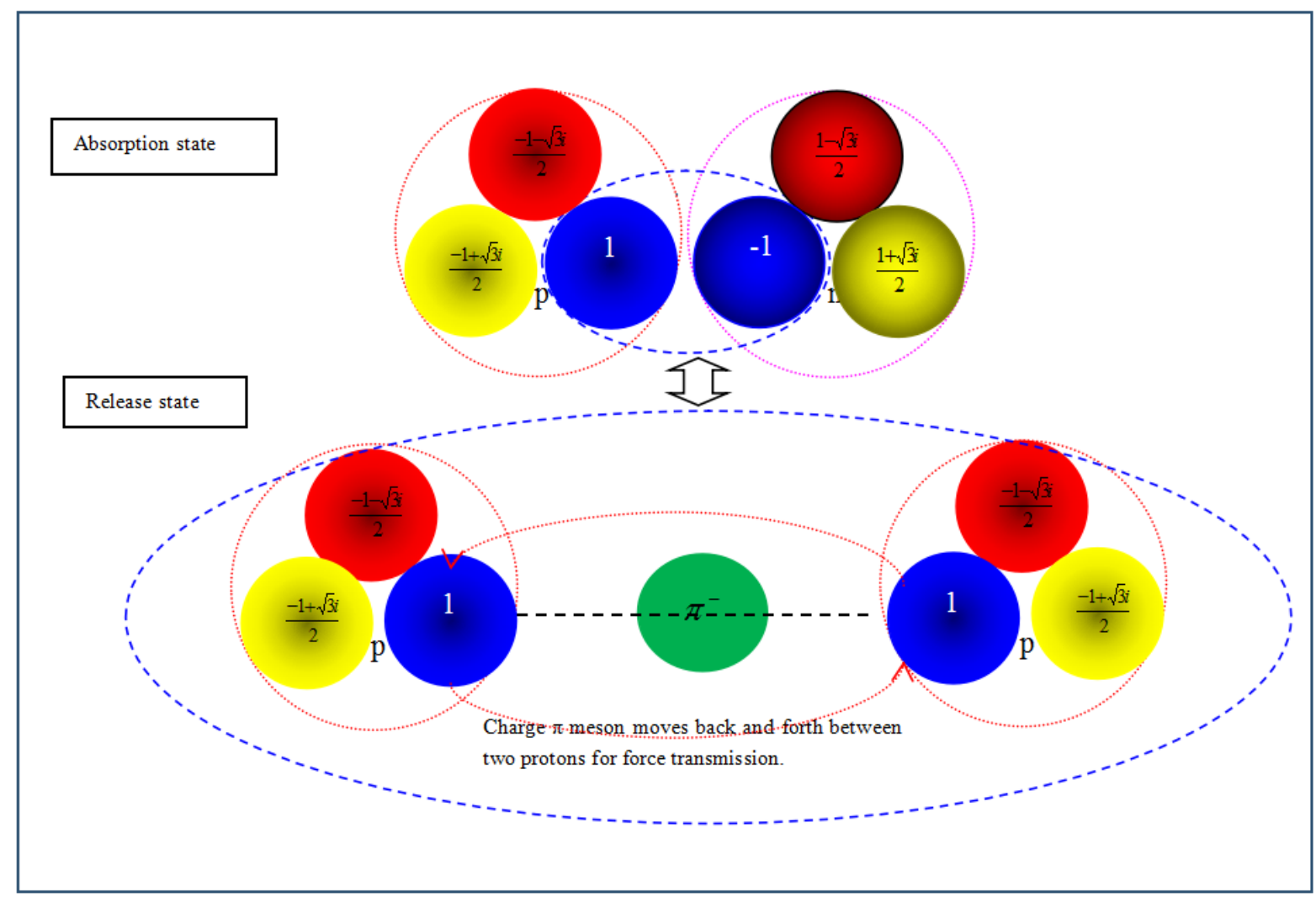

Figure 14. $\pi$ meson release and absorption states always exist in deuteron.

In consideration of continuous movement of $\pi$ meson between proton and neutron, the balance state in deuteron is namely the release state thereof. Under such state, two protons make circular movement around nucleus, and $\pi$ meson locates at the center of the central circle. The mechanical formula for such state can be established as follows:

$$
\begin{gathered}
2 m_{p} v r=\hbar \\
\frac{m_{p} v^{2}}{r}=\frac{\hbar c}{4 r^{2}}\left(\mathrm{e}^{-2(r / a-1)}+3 \alpha\right)
\end{gathered}
$$

The following formula is set:

$$
\left\{\begin{array}{l}
k=\frac{r}{a} \\
b=\frac{m_{p}}{m_{\pi^{ \pm}}(1-0.5 \ln (1+\alpha))}
\end{array}\right.
$$


The experimental values of proton, $\pi$ meson and fine structure constant can be put into the formula to find constant $b$.

$$
b=6.747
$$

Formulae (73), (74) and (75) are combined and simplified as follows:

$$
\frac{1 / 4}{k}\left[\mathrm{e}^{-2(k-1)}+3 \alpha\right]=\frac{1}{4 b}
$$

The potential energy formula of deuteron is as follows:

Kinetic energy of each nucleon is as follows:

$$
V(r)=-\frac{\hbar c}{2 r}\left(\mathrm{e}^{-2(r / a-1)}+3 \alpha\right)
$$

Formulae (76) and (77) are put into the following formula to obtain:

$$
\Delta E_{1}=V(r)-2 E=-5.152 \mathrm{MeV}
$$

Deuteron has a probability of $1 / 2$ respectively for two states-absorption state and release state. Under absorption state, the sum of the charges carried by protons and neutrons therein is zero, so there is no any interaction; protons and neutrons are respectively under free state, and the binding energy is also zero, and the binding energy of deuteron shall be the mean value under the two states.

$$
\Delta E=\frac{1}{2} \times E_{1}+\frac{1}{2} \times 0=-2.576 \mathrm{MeV}
$$

But the influence of the mass of $\pi$ meson located at the center is not considered in above formula. Notably, the mass of this $\pi$ meson is not a constant. The influence of two factors may be considered for the estimation thereof.

1) $\pi^{ \pm} \rightarrow \mathrm{e}+v^{\mathrm{e}}$ is true, so the mass of $\pi$ meson will not be lower than that of electron. Therein, the probability of $\pi$ meson under release state in deuteron is $2 / 3$ (internal quark composition of proton is (uud), $\pi$ meson can be only absorbed by $2 \mathrm{u}$ quarks to generate $\mathrm{d}$ quark; the probabilities for $\pi$ meson under absorption state and under release state are respectively $1 / 2 \star 2 / 3=1 / 3$ and $1-$ $1 / 3=2 / 3)$.

$$
E_{\pi^{ \pm}}>\frac{2}{3} E_{e}=0.3407 \mathrm{MeV}
$$

2) Influence of internal stress action inside deuteron on $\pi$ meson

$$
\Delta E_{\pi^{ \pm}}=E_{n}-E_{p}-\frac{1}{2} \Delta E=1.293-1.288=0.005 \mathrm{MeV}
$$

Formulae (82) and (83) are comprehensively considered to obtain the estimated mass of $\pi$ meson:

$$
E_{\pi^{ \pm}}=\frac{2}{3} E_{e}+\Delta E_{\pi^{ \pm}}=0.3457 \mathrm{MeV}
$$

Finally, the binding energy of deuteron is obtained as follows:

$$
\Delta E_{D \text { the }}=\Delta E+m_{\pi^{ \pm}}=-2.230 \mathrm{MeV}
$$

The experimental value is put into the formula: 


$$
\Delta E_{D \exp }=E_{D \exp }-E_{n \exp }-E_{p \exp }=-2.225 \mathrm{MeV}
$$

Error is as follows:

$$
\Delta=E_{D \exp }-E_{D \text { the }}=+0.005 \mathrm{MeV}
$$

Error rate is as follows:

$$
\eta=\left(\frac{\Delta E_{D \text { the }}}{\Delta E_{D \exp }}-1\right) \times 100 \%=\left(\frac{2.230}{2.225}-1\right) \times 100 \%=0.22 \%
$$

The theoretical value is basically consistent with the experimental value.

\subsection{Estimation of Binding Energy of Three Atomic Nucleuses}

Triton, He-3 and He-4 have complex internal spatial structure, so the binding energy can be only estimated.

He-3 has two protons and a neutron, when the neutron release a pion, the strong nuclear force will spread between three protons, Strong interaction of He-3 is equivalent to the strong interaction of three pairs of deuteron (as shown in Figure 13(c)), and the binding energy thereof is three times of that of deuteron. Considering the influence of the electrostatic potential difference, the binding energy of he- 3 can be obtained.

$$
\begin{aligned}
\Delta E_{\text {He-3the }} & =2 \Delta E_{D \text { the }}+\Delta E-\frac{(3 / 2+2) \alpha}{4 k} m_{\pi^{ \pm}}(1-0.5 \ln (1+\alpha)) \\
& =-2.230 \times 2-2.576-0.5136 \\
& =-7.550 \mathrm{MeV}
\end{aligned}
$$

Strong interaction of triton is similar with that of He-3 (as shown in Figure 13(b)), but it is necessary to consider the repulsive interaction between two protons in He-3, and its binding energy is slightly lower than that of He-3.

$$
\begin{aligned}
\Delta E_{\text {He-3the }} & =\Delta E_{\text {Dthe }}+2 \Delta E-\frac{(3 / 2+2) \alpha}{2 k} m_{\pi^{ \pm}}(1-0.5 \ln (1+\alpha)) \\
& =-2.230-2.576 \times 2-1.0272 \\
& =-8.411 \mathrm{MeV}
\end{aligned}
$$

The internal spatial structure of He-4 is a normal triangular pyramid (as shown in Figure 13(d)), and each face of the normal triangular pyramid is equivalent to the internal symmetrical structure of a He-3, so its binding energy is four times of that of He-3. Additionally, two faces are lack of $\pi$ mesons, so the influence of two pairs of proton-proton force must be considered. Specifically, the binding energy is measured and calculated as follows:

$$
\begin{aligned}
\Delta E_{\mathrm{He}-4 \text { the }} & =4 \Delta E_{\mathrm{He}-4 \text { the }}+2\left(\Delta E_{\mathrm{He}-4 \text { the }}-\Delta E_{T \text { The }}\right) \\
& =-7.550 \times 4+2 \times(7.550-8.411) \\
& =28.48 \mathrm{MeV}
\end{aligned}
$$

The comparison between the theoretical value and thee experimental value of the binding energy of three atomic nucleuses is shown in the following Table 2. 
Table 2. Binding energy Comparison Table for Three Atomic Nucleuses.

\begin{tabular}{ccccc}
\hline Particle Name & $\begin{array}{c}\text { Theoretical Value } \\
A \text { of binding } \\
\text { energy (MeV) }\end{array}$ & $\begin{array}{c}\text { Experimental } \\
\text { Value } B \text { of binding } \\
\text { energy }(\mathrm{MeV})\end{array}$ & Error $=A-B$ & $\begin{array}{c}\text { Error Rate }= \\
(A / B-1)^{\star} \%\end{array}$ \\
\hline He-3 & -7.550 & -7.718 & 0.168 & $-2.2 \%$ \\
Triton & -8.411 & -8.489 & 0.078 & $-0.92 \%$ \\
He-4 [7] & -28.48 & -28.30 & 0.16 & $-0.57 \%$ \\
\hline
\end{tabular}

Remarks: 1) The experimental value of He-3 binding energy cannot be found, so it is obtained through deducting the mass [1] of two neutrons and one proton from the mass [1] of He-3.2) The experimental value of triton cannot be found, so it is obtained through deducting the mass [1] of two neutrons and one proton from the mass [1] of triton.

According to the comparison between the theoretical value and the experimental value of the binding energy of three atomic nucleuses, we find significant error and high error rate, but we cannot deny that such thought can be used for analyzing the internal nucleus action of atomic nucleuses, thus presenting important reference value.

\section{7. $\Delta(1232)$ Strong Interaction}

\section{1. $\Delta(1232)$ Internal Symmetrical Structure}

$\Delta$ (1232) particles family includes four types of particles, respectively $\Delta++$ (1232), $\Delta+$ (1232), $\Delta 0$ (1232) and $\Delta-$ (1232). $\Delta$ (1232) particles family plays a special role, and forms the complete baryon family of $\mathrm{u}$ and $\mathrm{d}$ quark structure together with protons and neutrons. According to the mass information analysis, $\Delta$ (1232) particles family, different from proton and neutron which are usually directly composed of quarks, is composed of proton or neutron and two $\pi$ mesons. In other words, the strong interaction thereof belongs to baryon-meson strong interaction.

$\Delta$ (1232) particles family has internal odd symmetrical structure, as the same as the internal symmetrical structure of proton and neutron (as shown in Figure $15)$.

\section{2. $\Delta$ (1232) Mass Ratio and Mass Estimation}

$\Delta$ (1232) internal symmetrical structure can be also described by Dirac equation, but the specific process is similar with the process for the mentioned particles, so it will not be repeatedly explained in this chapter.

Theoretical values of the masses of the four types of particles are as follows:

$$
\begin{aligned}
m_{\Delta++}: m_{\Delta+}: m_{\Delta 0}: m_{\Delta-}= & \left(1+0.5 \ln \left(1-\frac{2(3-\sqrt{3})}{3} \alpha\right)\right)^{-}:\left(1+0.5 \ln \left(1-\frac{2}{3} \alpha\right)\right)^{-} \\
& :\left(1+0.5 \ln \left(1-\frac{2}{3} \alpha\right)\right)^{-}:\left(1+0.5 \ln \left(1-\frac{2}{3} \alpha\right)\right)^{-} \\
= & 1.00066: 1: 1: 1
\end{aligned}
$$




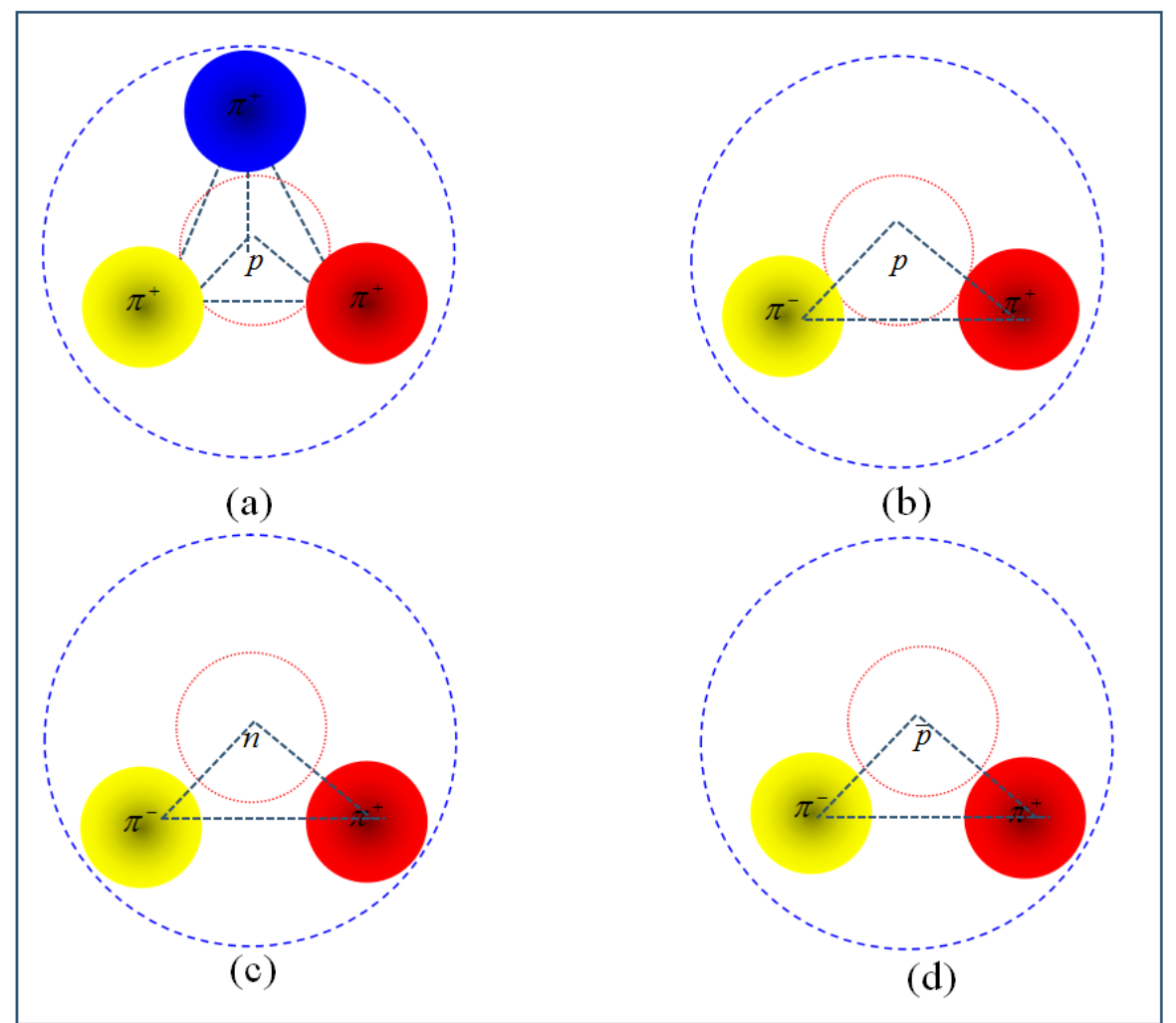

Figure 15. (a) $\Delta++$ (1232) Internal odd symmetrical structure; (b) $\Delta+$ (1232) Internal odd symmetrical structure; (c) $\Delta 0$ (1232) Internal odd symmetrical structure; (d) $\Delta-$ (1232) Internal odd symmetrical structure.

Table 3. $\Delta$ (1232)'s mass comparison table.

\begin{tabular}{lcccc}
\hline $\begin{array}{c}\text { Particle } \\
\text { Name }\end{array}$ & Mass Formula & $\begin{array}{c}\text { Theoretical } \\
\text { Value } A \text { of } \\
\text { Mass }(\mathrm{MeV})\end{array}$ & $\begin{array}{c}\text { Experimental } \\
\text { Value }[2] B \text { of } \\
\text { Mass }(\mathrm{MeV})\end{array}$ & $\begin{array}{c}\text { Error Rate }= \\
(A / B-1)^{*} \%\end{array}$ \\
\hline$\Delta_{++}$ & $m_{\Delta++}=m_{0}\left(1+0.5 \ln \left(1-\frac{2(3-\sqrt{3})}{3} \alpha\right)\right)^{-}$ & 1221 & $1232 \pm 2$ & $-0.89 \%$ \\
$\Delta_{+}$ & $m_{\Delta+}=m_{0}\left(1+0.5 \ln \left(1-\frac{2}{3} \alpha\right)\right)^{-}$ & 1220 & $1232 \pm 2$ & $-0.97 \%$ \\
$\Delta_{0}$ & $m_{\Delta 0}=m_{0}\left(1+0.5 \ln \left(1-\frac{2}{3} \alpha\right)\right)^{-}$ & 1220 & $1232 \pm 2$ & $-0.97 \%$ \\
$\Delta_{-}$ & $m_{\Delta-}=m_{0}\left(1+0.5 \ln \left(1-\frac{2}{3} \alpha\right)\right)^{-}$ & & & \\
\end{tabular}

The experimental values of four types of $\Delta$ (1232) particles are all $1232 \pm 2$ $\mathrm{MeV}$, and the specific value thereof is basically as the same as Formula (92). $m_{0}=m_{p}+2 m_{\pi \pm}$ is assumed to estimate the masses of the four types of $\Delta$ (1232) particles, and the estimated value is compared with the experimental value (as shown in Table 3).

The theoretical value is basically consistent with the experimental value. 


\section{Strong Interaction of $\eta$ Particle and $\eta^{\prime}(958)$ Particle}

Internal triangular-pyramid symmetrical structure of $\eta$ particle is as shown in Figure 16(a); internal dual triangular-pyramid symmetrical structure of $\eta^{\prime}(958)$ particle is as shown in Figure 16(b).

$\eta$ particle has internal triangular-pyramid symmetrical structure and is composed of four $\pi$ mesons which generate inter-meson type strong interaction. $\eta^{\prime}$ particle has dual triangular-pyramid symmetrical structure combined by two $\eta$ particles, and is composed of seven $\pi$ mesons which generate inter-meson type strong interaction. Without considering electrostatic force, the mass of the two types of particles is namely the sum of the mass of $\pi$ mesons included therein.

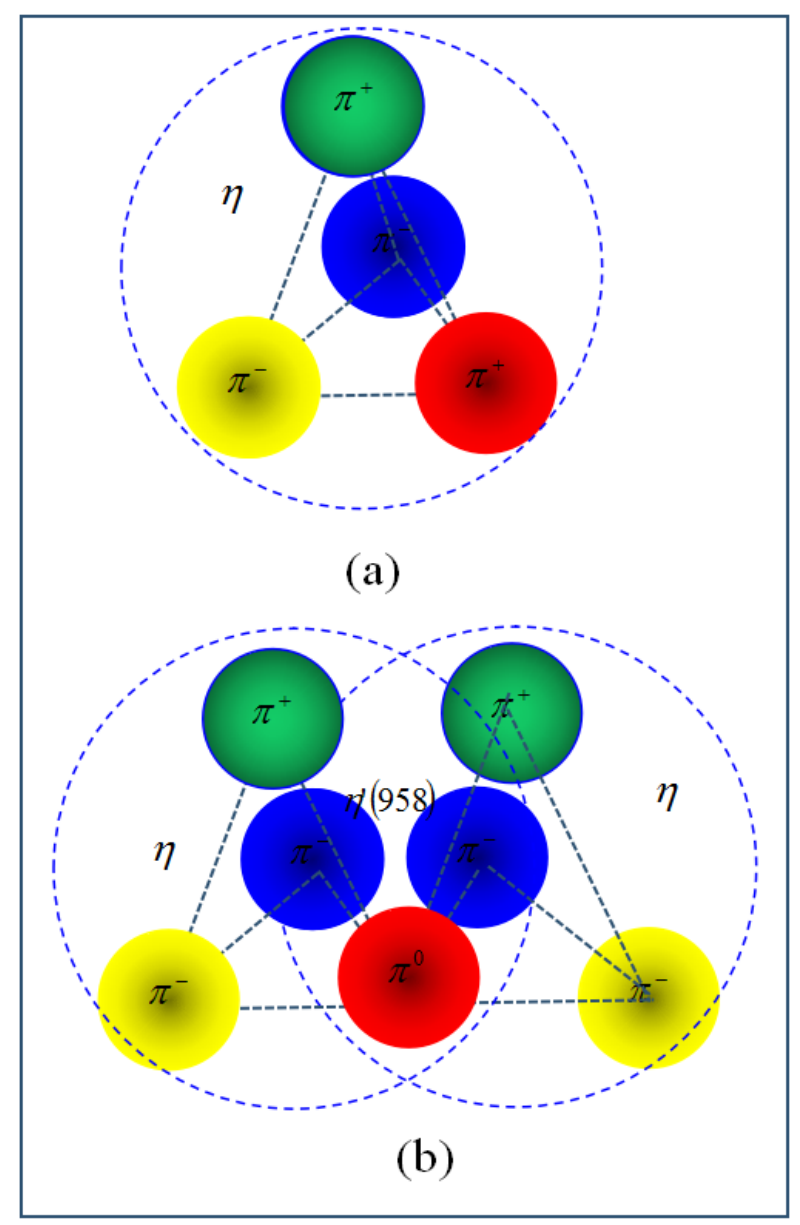

Figure 16. (a) Internal triangular-pyramid symmetrical structure of $\eta$ particle; (b) Internal dual triangular-pyramid symmetrical structure of $\eta^{\prime}(958)$ particle.

Table 4. $\Delta$ (1232)'s mass comparison table.

\begin{tabular}{ccccc}
\hline Particle Name & Mass Formula & $\begin{array}{c}\text { Theoretical Value } A \\
\text { of Mass }(\mathrm{MeV})\end{array}$ & $\begin{array}{c}\text { Experimental Value } B \\
\text { of Mass }(\mathrm{MeV})\end{array}$ & $\begin{array}{c}\text { Error Rate } \\
=(A / B-1)^{*} \%\end{array}$ \\
\hline$\eta$ & $m_{\eta}=2\left(m_{\pi \pm}+m_{\pi 0}\right)$ & 549.09 & $547.75 \pm 0.12$ & $0.24 \%$ \\
$\eta^{\prime}(958)$ & $m_{\eta}=3 m_{\pi \pm}+4 m_{\pi 0}$ & 958.61 & $958.78 \pm 0.14$ & $-0.017 \%$ \\
\hline
\end{tabular}


The theoretical value is basically consistent with the experimental value in Table 4.

\section{Summary}

As implied by the title of the paper- "Research on Strong Interaction", the paper aims at solving strong force potential formula.

Firstly, the strong interaction is globally divided into five different types according to different stress particles, and 12 types of different representative particles are selected for different types of strong interactions, thus presenting the comprehensiveness of the research objects.

The first part of the main text is the hypothesis for strong force potential. Charge type of strong force, relation between strong force intensity and fluctuation, hadron state and its limitation cause are researched to propose reasonable strong force potential formula, but the applicability of strong force potential formula shall be verified under specific particle state. Two tools are needed for solving particle state, namely: internal symmetrical structure diagram of research object, which is used for preliminarily integrating strong force potential and electrostatic potential operators; Dirac equation which is undoubtedly successful for solving high-energy state of particles. Strong force potential and electrostatic potential operators preliminarily integrated are put into Dirac equation to improve solution efficiency.

The second part of the main text is the verification of the applicability of strong force potential formula. The ground state function for the charged particle can be obtained through Dirac equation description, but the most effective evidence for this part is the theoretical value of the mass ratio of charged particle and neutral particle, and it is greatly accurate to compare the theoretical value of the mass ratio with the corresponding experimental value to judge a series of mentioned processes and hypotheses.

Error or error rate between the theoretical values of the mass ratios of 10 particles and the corresponding experimental values is very small (the error rate between the theoretical values of $\eta$ particle and $\eta^{\prime}(958)$ particle and the corresponding experimental values is very small). In other words, the theoretical value is consistent with the experimental value, thus proving the validity of strong force potential formula. Hadron state or the state for forming stable structure between hadrons is described by state function which can meet Formula (36).

By observing the strong interaction law of the above 12 hadrons, we found that all the strong interactions have the participation of meson (pion or kaon), which is the medium of strong nuclear force. It is spreading the role of power between quarks, Mesons, baryons, Meson and baryon, so the meson is not only the stress particles, but also the media.

\section{References}

[1] CODATA Internationally Recommended 2014 Value of the Fundamental Physical 
Constants. http://physics.nist.gov/cuu/Constants/index.html

[2] Amsler, C., et al., Particle Data Group. (2017) The Review of Particle Physics. Chinese Physics C, 40, 100001. http://pdg.lbl.gov

[3] Eidelman, S., et al., Particle Data Group. (2004) Physics Letters B, 592, 1. http://pdg.lbl.gov

[4] Dirac, P.A.M. (1928) The Quantum Theory of the Electron. Proceedings of the Royal Society of London. Series A, Containing Papers of a Mathematical and Physical Character, 117, 610-624. http://www.jstor.org/stable/94981 https://doi.org/10.1098/rspa.1928.0023

[5] Dirac, P.A.M. (1930) A Theory of Electrons and Protons. Proceedings of the Royal Society A: Mathematical, Physical and Engineering Sciences, 126, 360. https://doi.org/10.1098/rspa.1930.0013

[6] Huang, Z.D. (2017) The Research on Relationship between Neureinos and Weak Force. Modern Physics, 7, 197-212. https://doi.org/10.12677/mp.2017.75023

[7] Nogga, A., Bogner, S.K. and Schwenk, A. (2004) Physical Review C, 70, Article ID: 061002. 